\title{
Polymers and nano-objects, a rational combination for developing health monitoring biosensors
}

\author{
Michael Holzinger*, Paulo Henrique M. Buzzetti, and Serge Cosnier *
}

Univ. Grenoble Alpes, DCM UMR 5250, F-38000 Grenoble, France. CNRS, DCM UMR 5250, F-38000

Grenoble, France

\begin{abstract}
Biosensors are essential tools in the health and the environmental sectors since they allow fast and reliable diagnostics and analysis. Biosensors are defined by the biological sensing element, which contains biomolecules or synthetic, bioinspired entities with unique specificity towards the analyte. The biological or bioinspired recognition even can be transduced into an electric, electrochemical, or optical signal. A constant challenge for the development of biosensors is the confinement of these selective entities in devices without altering the biological activity. Polymers are great candidates to serve as substrates for the immobilization or entrapment of bioreceptors related to well established synthesis routes with highly modulable chemical functions to create an almost ideal environment for the biomolecules. Nanomaterials are of constant increasing interest since they allow not only to increase drastically the specific surface for higher amounts of receptor units, but also provide electric or optical properties leading to enhanced signal capture. As for bioreceptors, such nanomaterials can be integrated polymer matrices for reliable processability. This review aims to summarize selected original examples about biosensors for health monitoring using beneficial combinations of nanomaterials and polymers.
\end{abstract}

\section{Introduction}

Monitoring risk factors of patients or medical screening of the population is essential for early detection and control of diseases. The strong evolution in this field led to portable, miniaturized devices with wireless data transmission systems to improve comfort and treatments [1]. Very prominent became point of care tests where patients can monitor the pathogen at home without the need of qualified staff or clinical laboratories [2]. To analyse biological parameters like blood sugar, cancer or cardiovascular markers, biosensors are the most efficient devices related to their capacity to capture one specific analyte in complex body fluids $[3,4]$. The sensing entities are generally enzymes [5], proteins of immune systems [6], DNAs [7], or synthetic nucleotides like aptamers [8] or peptides like DARPins [9] depending on the type of disease.

One important factor is the choice of the substrate for optimal immobilization of the bioreceptors and efficient transduction of the recognition event. Historically, the first biosensors for health monitoring was an electrochemical glucose sensor, where the enzyme glucose oxidase was trapped in a polyacrylamide gel [10]. Polymers became the material of choice for the development of biosensors since properties and chemistry can easily be tuned and customized for each receptor type [11]. Particular interesting are conductive polymers which can facilitate electron transfers with enzymes, change the electronic signature and thus serve as transducer element [12]. Such polymers can directly be generated on electrodes via electropolymerization which allows the control of morphology, thickness and spatial addressing of polymer whatever size and the form of conducting surface [13].

More recently, nanomaterials emerged in the field of biosensors developments [14, 15]. The global advantage of nanomaterials is the high specific surface that allows the immobilization of more bioreceptor units leading consequently to improve performances in terms of sensitivity and limit of 
detection. When reduced to the nanoscale, some materials change their properties with the appearance of intrinsic quantum effects which lead to particular optical, electrical and mechanic features [16]. Due to their unique physicochemical properties, nanoparticles, in particular metal and metal oxide nanoparticles, have been widely exploited for health and medical applications [17-20]. Since nanomaterials are in the same size range as biomolecules used for biosensors, the immobilization or confinement is as important. Polymers play again a crucial role for the processing of nanomaterials and this led to the vast research topic of nanocomposites [21, 22].

Nanocomposites can be defined as a material formed from at least two components and where at least one is a nanosized material. Such nanofillers can be of 0 (spheres), 1 (tubes, fibres) and 2 (layered sheets) dimensions and be of any imaginable chemical nature. In this context, biomacromolecules used as receptor units in biosensors can thus be considered as nanomaterial where the first bio-nanocomposite was formed in 1967. However, the researchers Roy and Komarneni used firstly term nanocomposite describing sol-gel processes in the period 1982-1983 [23, 24].

Nanocomposites revolutionized the vast topic of health care research and are present in various applications concerning gene therapy, drug delivery, tissue engineering, food preservation, bioimaging, and biosensing [25]. Tremendous advances were reported and reviewed in the biomedical diagnostic sector about high performing sensors and biosensors with detection limits down to individual molecules using polymer nanocomposites [26, 27].

In the following section, a selection of original approaches for the combination of nanomaterials and polymers for healthcare biosensors is presented.

\section{Imprinted Polymer -Quantum dots (QDs):}

Quantum dots (QDs) are semiconducting nanoparticles with particular optical properties. Their narrow emission spectrum depends on the particle size influencing the band gap and thus the emission wavelength [28]. Quantum dots are therefore of interest for optoelectronics [29] but also play an important role in the biomedical field like bioimaging, drug delivery, or sensing [30]. However, the most studied semiconductor material is based on cadmium chalcogenides which represents toxicological issues [31]. Zn based QDs [32]and carbon QDs [33] represent less toxic alternatives but the obtained QDs and, in particular, carbon QDs, still lack of homogeneity for defined narrow emission spectra. The quantum efficiency and synthesis yields are also still to improve [34]. For optical biosensing, the transduction principle is generally based on non-radiative energy transfer between the excited QDs and an acceptor such as gold nanoparticles [35] or graphene [36]. QDs and acceptors are therefore used as labels and need relative complex preparation and synthesis procedures to construct the sensing element. An elegant solution is when the analyte itself can act as acceptor or quenching unit. One quite original strategy is to coat the QDs with molecular imprinted polymers (MIPs) which serves as specific receptor. MIPs are formed by polymerization of appropriate monomers like silicates, silanes, or organic monomers in presence of the target analyte. After the polymerization reaction, the target molecule is extracted leaving a specific imprint on the polymer. With the resulting MIP-QD composite, the measured quenching effect during the recognition events in the evaluated target solutions allows to quantify the analyte concentration [37]. The different quenching mechanisms and a series of examples are nicely reviewed in references $[38,39]$.

This detection principle is very efficient for small molecules like drugs or biomarkers but can also be applied to biomacromolecules like proteins.

For instance, Wang et al. used phycocyanin as model protein to imprint polydopamine on thioglycollic acid and glutathione capped QDs [40]. This biosourced polymer is presented as very appropriate polymer for protein imprints. The phycocyanin concentration dependent quenching provided a nicely linear profile and detection limits in the sub micromolar range could be obtained 
and was validated with phycocyanin spiked sea and lake waters. To demonstrate the suitability of this setup, bovine haemoglobin was used as template leading to similar biosensor performances.

Related to the electroactivity of dopamine, a similar setup with electrochemical transduction was reported. Mo et al. electropolymerized dopamine on glassy carbon electrodes in presence of squamous cell carcinoma antigen (SCCA), the target analyte. SCCA is a $48 \mathrm{kDa}$ glycoprotein and a useful tumor marker for the diagnosis of lung cancer. ZnSe quantum dots, attached to a metal organic framework (MOF) and modified with anti-SCCA antibodies. After rebinding of SCCA on the polymer in the analyte solution, the antibody MOF QDs are immobilized on the electrode surface leading to an electrochemiluminescent signal. The MOF allowed an improved efficiency to reduce the sacrificial electron acceptor $\mathrm{S}_{2} \mathrm{O}_{8}{ }^{2-}$ and thus an improved electrochemiluminescent signal. With this configuration, a stable and reproducible SCCA biosensor was obtained [41].

Another elegant strategy was firstly reported by $\mathrm{Li}$ et al. This team used the epitope phenylphosphonic acid as printing template for the detection of tyrosine phosphopeptide [42]. In this work, a "turn on" concept was developed which is based on a passivation of "trap-states" in the MIPQD composite after capture of phenylphosphonic acid or tyrosine phosphopeptide leading to enhanced fluorescence.

A more sophisticated setup for the same analyte was proposed by Saeedzadeh Amiri and colleagues [43]. Here, two optical probes with different emission wavelengths were used to detect phenylphosphonic acid or tyrosine phosphopeptide via colour changes since the target quenched the emission of the QDs with red emissions but not the one with green emission. Furthermore, a mesoporous structure was formed on the outer shell of the MIP using the amphiphile ionic liquid 1tetradecyl-3-methylimidazolium chloride. The microwave assisted polymerization of the silane in presence of these ionic liquid micelles and the epitope led to a porous structure with enhanced receptor sites (Figure 1). This biosensor design had excellent regeneration capacities and led to a 3 orders of magnitude improvement for the detection limit compared to the precedent setup.

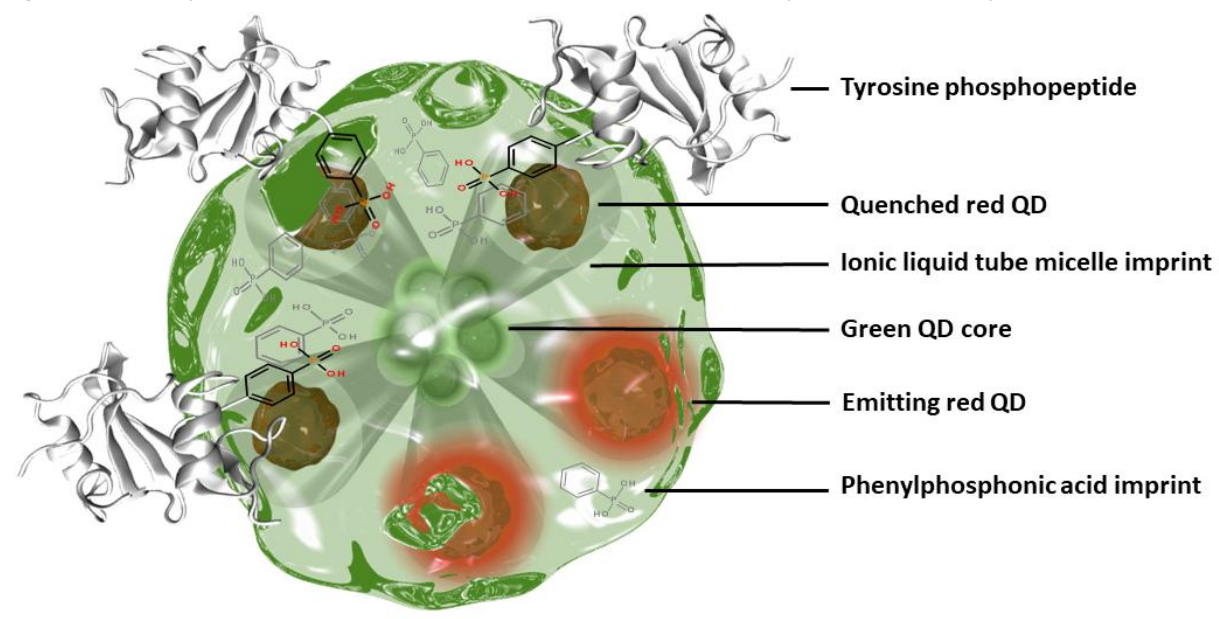

Figure 1: Illustration of a phenylphosphonic acid imprinted nanoparticle with a green light emitting QD core and a porous quenchable red light emitting QD shell during recognition of tyrosine phosphopeptide or phenylphosphonic acid.

\section{Polymer Gold NP}

Gold nanoparticles are by the mostly used material for analytics and diagnosis related to the combination of excellent conductivity and optical properties [44-46]. In particular, the plasmonic behaviour of nanosized gold particles made this material the component of choice for analytical SPR and Raman setups. The localized resonant plasmons are extremely sensitive to their environment and can interact with surface plasmons on macroscopic surfaces leading to an amplification of the signals [47]. By eliminating bulk effects from classic SPR setups, localized plasmon resonance allows higher selectivity and does not need sophisticated equipment [48]. However, the sensitivity and 
analytical performances are generally lower. This issue can be improved by controlling the spatial distribution and spacing between the gold nanoparticles to optimize the plasmonic properties [49]. One interesting approach is the use of block copolymers forming self-organized nanopatterns to obtain an array of homogeneously distributed gold nanoparticles. Lu et al. used poly(styrene-b-4vinylpyridine) block copolymer to form a brush layer on glass fibres [50]. The exposed vinylpyridine patterns allowed to form dense gold nanoparticle array with controlled distances and particle sizes without aggregates and vacancies. The authors then formed an optical immunosensor for the detection of human IgG with immobilized goat anti-human IgG on the glass fibre AuNP array (Figure 2). The immune response was monitored in real time by measuring changes in resonance wavelength and intensity with a standard fibre-coupled spectrophotometer. The obtained detection limit was in the same order of magnitude as the detection limit using a prism-based SPR instrument.

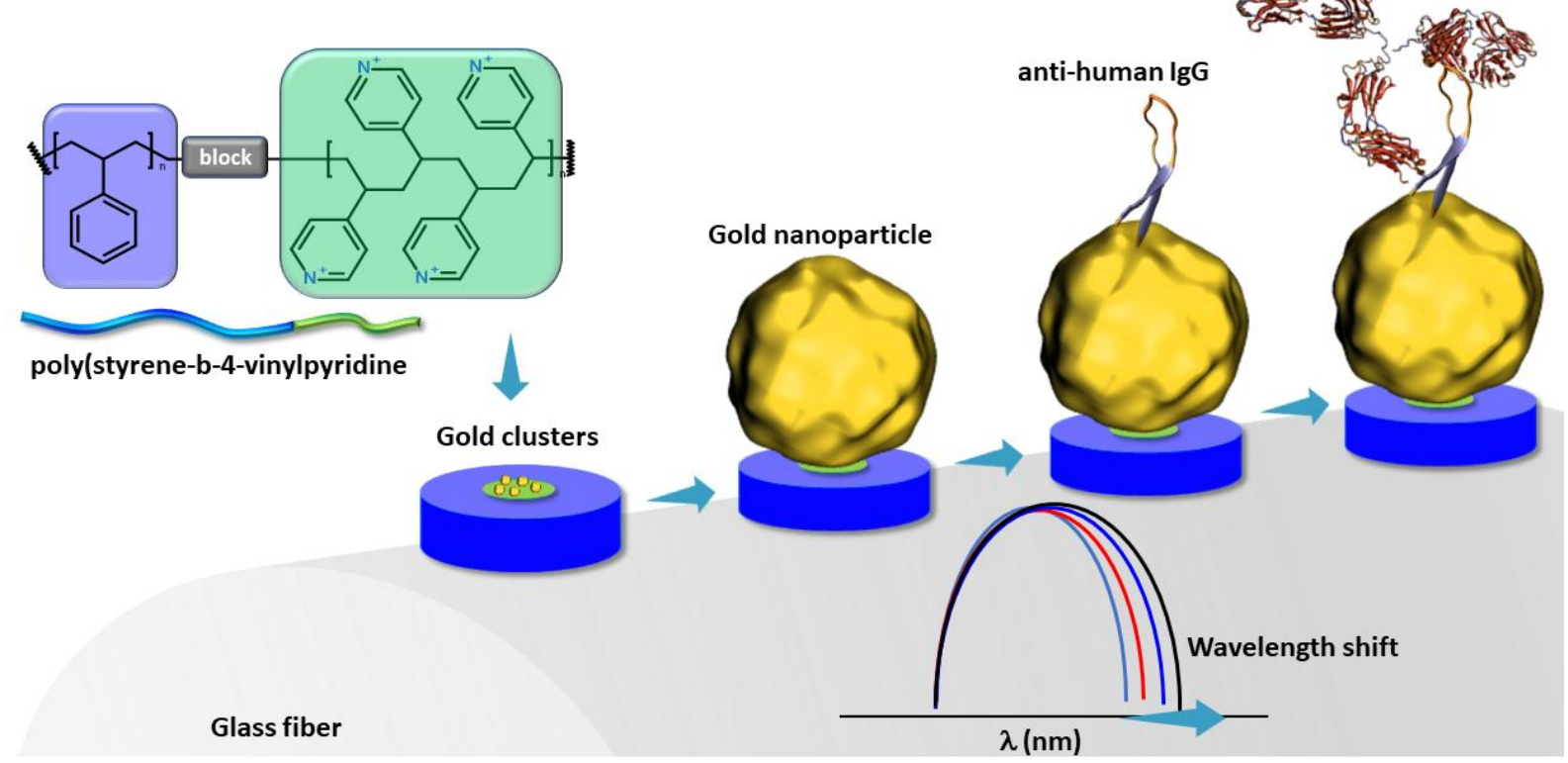

Figure 2: Schematic presentation of the self-assembled patterning of a glass fiber using poly(styrene-b-4vinylpyridine) block copolymer for spatial controlled growth of gold nanoparticles and the formation of a human IgG biosensors using localized plasmon resonance.

Less sophisticated but as efficient patterns are necessary for electrochemical transduction. Avelino et al. formed a flexible DNA sensor using an electropolymerized polypyrrole layer on ITO modified polyethylene terephthalate [51]. Polydisperse gold nanoparticles were electro synthesized on the polypyrrole film, functionalized with a designed oligonucleotide for the recognition of families of human papillomavirus in cervical specimens. In this setup, the gold nanoparticles served as amplifier for the cyclic voltammetric and impedimetric signals due to their good conductivity and enhanced surface area. The detection limit covers the realistic range of infected persons. This setup could therefore be validated with clinical samples, which also confirmed the excellent specificity of the designed oligonucleotide.

One important point in health monitoring is the environmental impact of the devices since they have to be littered after direct contact with patients. Toxic or polluting components are therefore to avoid. Vural et al. developed a disposable and cost-effective sensing element based on pencil graphite electrodes which were modified with synthesized gold nanoparticle / peptide nanotube composites [52]. The conductive polymer polyaniline was then electropolymerized as thin film around the deposited composite. This setup served to detect prostate specific antigen (PSA) via amperometry. The electrochemical signal was obtained by formation of sandwich structures with anti-PSA antibody as bioreceptor unit, which capture the target PSA. The final layer was a secondary anti-PSA antibody labeled with horseradish peroxidase, which reduced hydrogen peroxide to water via hydroquinone 
mediated electron transfer with the electrode. The obtained detection limit is suitable for medical applications.

Table 1 summarises the performances of the discussed biosensors setups based on the combination of polymers and nanomaterials. Considering the different analytes and transduction techniques, it is difficult to compare the reported values. Nonetheless, a clear tendency to higher performances with more sophisticated systems can be observed.

Table 1: biosensor performances using polymer nanoparticle assemblies

\begin{tabular}{|c|c|c|c|c|c|}
\hline Polymer & Template / Analyte & Nanomaterial & $\begin{array}{l}\text { Limit of } \\
\text { detection }\end{array}$ & $\begin{array}{l}\text { Linear } \\
\text { range }\end{array}$ & ref \\
\hline Polydopamine & Phycocyanin & $\begin{array}{l}\text { CdTe QDs capped } \\
\text { with thioglycollic } \\
\text { acid and } \\
\text { glutathione } \\
\end{array}$ & $0.069 \mu \mathrm{mol} \mathrm{L}^{-1}$ & $\left.\begin{array}{c}0.3-5.0 \\
(\mu \mathrm{mol} \mathrm{L} \\
-1\end{array}\right)$. & [40] \\
\hline Polydopamine & $\begin{array}{c}\text { Squamous cell } \\
\text { carcinoma antigen }\end{array}$ & $\begin{array}{c}\text { ZnSe QDs } \\
\text { attached to MOF }\end{array}$ & $31 \mathrm{fg} \mathrm{mL}^{-1}$ & $\begin{array}{c}0.0001- \\
100 \\
\left(\mathrm{ng} \mathrm{mL}^{-1}\right)\end{array}$ & [41] \\
\hline $\begin{array}{c}\text { 1-[3-(trimethoxysilyl) } \\
\text { propyl] urea cross linked } \\
\text { with } \\
\text { octyltrimethoxysilane }\end{array}$ & $\begin{array}{l}\text { Phenylphosphonic } \\
\text { acid / tyrosine } \\
\text { phosphopeptide }\end{array}$ & $\begin{array}{c}\text { silica-capped } \\
\text { CdTe QDs }\end{array}$ & $0.37 \mu \mathrm{mol} \mathrm{L}^{-1}$ & $\begin{array}{c}0.5-35 \\
\left(\mu \mathrm{mol} \mathrm{L}{ }^{-1}\right)\end{array}$ & [42] \\
\hline $\begin{array}{c}\text { Propyltrimethoxysilane- } \\
\text { 3-methylimidazolium } \\
\text { chloride cross-linked } \\
\text { with 3- } \\
\text { Aminopropyltriethoxy } \\
\text { silane }\end{array}$ & $\begin{array}{l}\text { Phenylphosphonic } \\
\text { acid / tyrosine } \\
\text { phosphopeptide }\end{array}$ & $\begin{array}{c}\text { 4-mercapto } \\
\text { butyric acid } \\
\text { capped CdTe QDs } \\
\text { ( } 2 \text { sizes, } \\
2 \text { emission } \\
\text { wavelengths) } \\
\end{array}$ & $34 \mathrm{nmol} \mathrm{L}^{-1}$ & $\begin{array}{l}0.07-230 \\
\left(\mu \mathrm{mol} \mathrm{L}{ }^{-1}\right)\end{array}$ & [43] \\
\hline $\begin{array}{l}\text { Poly(styrene-b-4- } \\
\text { vinylpyridine) block } \\
\text { copolymer }\end{array}$ & Human IgG & $\begin{array}{c}\text { gold nanoparticle } \\
\text { array }\end{array}$ & $0.8 \mathrm{nmolL}^{-1}$ & - & [50] \\
\hline Polypyrrole & HPV genotypes & $\begin{array}{c}\text { gold } \\
\text { nanoparticles }\end{array}$ & $0.89 \mathrm{pg} \mu \mathrm{L}^{-1}$ & $\begin{array}{l}0.1-100 \\
\left(p g \mu L^{-1}\right)\end{array}$ & [51] \\
\hline Polyaniline & $\begin{array}{c}\text { Prostate specific } \\
\text { antigen }\end{array}$ & $\begin{array}{c}\text { peptide } \\
\text { nanotube-gold } \\
\text { nanoparticle }\end{array}$ & $0.68 \mathrm{ng} \mathrm{mL}^{-1}$ & $\begin{array}{c}1-100 \\
\left(\mathrm{ng} \mathrm{mL}^{-1}\right)\end{array}$ & [52] \\
\hline
\end{tabular}

For repeatable production of such analytical tools, however, such complex assemblies are less suitable for industrial application where simplified protocols are generally preferred to reduce costs and to assure reliability. The examples using low cost and environmental friendly materials combined with original but abridged fabrication procedures take into account such industrial needs. But, this should not prevent academic research to explore new principles despite the complexity at short term.

\section{Polymer based functional organic nanoparticles.}

Beside metallic nanoparticles, considerable efforts have been made in the development of organic nanoparticles based on self-assembly such as micelles, liposomes or polymersomes [53, 54]. Among then, biocompatible carbohydrate-coated nanoparticles have recently attracted a wide attention for biosensing and bioconversion applications. In particular, the synthesis of amphiphilic glycopolymers based on the coupling of hydrophilic poly-saccharides and hydrophobic synthetic polymers such as polystyrene has enabled the design of various structures (micelle, vesicle, worm-like micelle, nanoparticle) by self-assembly of these block copolymers $[55,56]$. Among the oligosaccharides, 
cyclodextrins as a hydrophilic block with a hydrophobic internal cavity can form inclusion complexes with a wide range of poorly water-soluble compounds. Thanks to these host-guest interaction properties of the nanoparticle shell, these nanoparticles provide a versatile platform that has been exploited for medical applications such as drug delivery [57].

In particular, the self-assembly of a $\beta$-cyclodextrin-polystyrene copolymer leads to spherical and stable glyconanoparticles (NP- $\beta C D$ ) in aqueous medium, which present an outer layer composed of $\beta$-cyclodextrin for post-functionalization by host-guest interactions. NP- $\beta C D$ have thus permit entrapment of hydrophobic fluorescent molecules in aqueous solution such as tetrazinenaphthalimide or redox mediators such as quinone and thiazoline, for the electrical wiring of enzymes [58-60] (Figure 3).

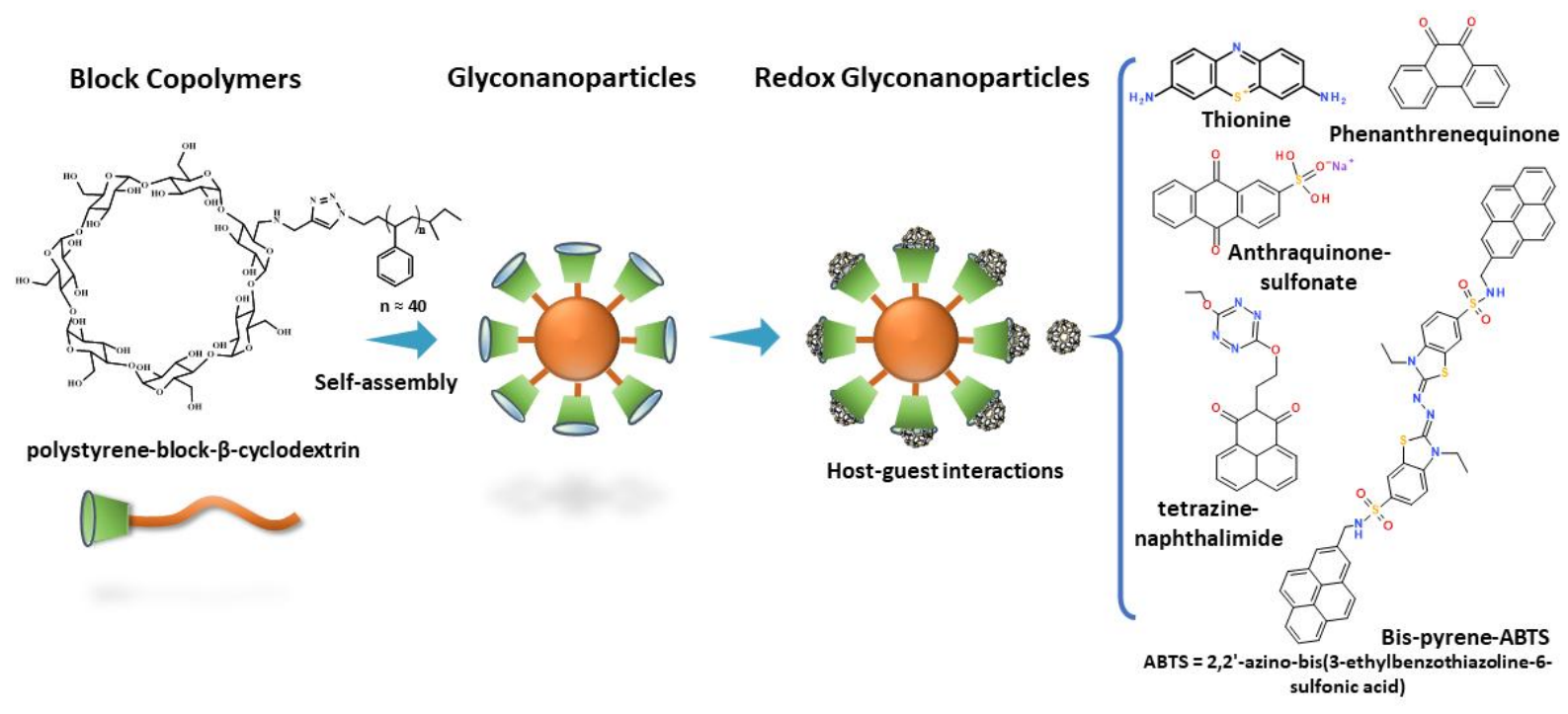

Figure 3: Formation of a biosourced block copolymer nanoparticle with $\beta$-cyclodextrin shell and its capacity to form inclusion complexes with a variety of redox molecules for the wiring of enzymes.

As previously reported for $\beta$-cyclodextrin-modified gold nanoparticles [61], the possibility of immobilizing the glyconanoparticles on a surface was studied via the electrogeneration of an anchoring polymer on an electrode. Thus, the immobilization of NP-BCD was carried out on an electropolymerized film of poly (pyrrole-adamantane) thanks to the affinity interactions between $\beta$ cyclodextrin and adamantane. Owing to the high affinity of biotin to form inclusion complexes with $\beta$-cyclodextrin [62], the immobilized NP- $\beta C D$ layer was applied to the anchoring of biotinylated enzymes to develop amperometric biosensor. Biotinylated glucose oxidase was thus anchored onto the NP- $\beta C D$-poly (pyrrole-adamantane) film for the detection of glucose via the electro-oxidation of $\mathrm{H}_{2} \mathrm{O}_{2}$ at the underlying platinum electrode. It should be noted the amperometric detection performance was improved by electropolymerizing a less hydrophobic polymer via the copolymerization of pyrrole-adamantane and 11-(1-pyrrolyl)undecanol increasing the permeation of $\mathrm{H}_{2} \mathrm{O}_{2}$ to the underlying platinum surface.

With the aim of developing new enzymatic labels for immunosensors or aptasensors, nanoparticles can be functionalized by enzymes and antibodies or antigens previously modified by adamantane or biotin groups. In this context, the density of the host-guest interaction sites of the nanoparticle shell must be reduced to avoid generating too many bonds between the nanoparticle and a protein. Indeed, the latter could induce the blocking of the conformational mobility of the enzyme and the creation of steric constraints, which would decrease the enzymatic activity. In order to modulate the density of $\beta$-cyclodextrin at the surface of the nanoparticles, hybrid nanoparticles have been produced by self-assembly of maltoheptaose-polystyrene and $\beta$-cyclodextrin-polystyrene in different proportions. Taking into account that maltoheptaose-polystyrene provides regular nanoparticles [63], as expected, its association with $\beta$-cyclodextrin-polystyrene also leads to stable and spherical 
nanoparticles with diameters ranging from 30 to $40 \mathrm{~nm}$ [64]. The host-guest interaction capacity of the hybrid nanoparticles was evaluated by cyclic voltammetry using the inclusion of a water-soluble redox probe: anthraquinone sulfonate. It appears that the intensity of the electroactivity of the hybrid nanoparticles is inversely proportional to the percentage of maltoheptaose-polystyrene present in the nanoparticle composition. This effect corroborates the possibility to modulate the anchoring properties of nanoparticles

Another type of nanoparticles based on a polymer shell surrounding a protein was described by $M$. Yan et al in 2010 for the intracellular delivery of proteins [65]. Three enzymes, HRP, Caspase 3 and superoxide dismutase were thus trapped in a polymeric shell, the diameter of the resulting nanocapsules being three times greater than the diameter of the native enzymes. The concept of manufacturing nanoparticles based on single protein consists in chemically grafting acrylic acid ( 5 to 10 units per protein) onto a protein and then using this functionalized protein as a template for the polymerization of vinyl monomers. This polymerization process leads to a crosslinked polymer cage anchored on the protein, described by the authors as a nanocapsule.

Following a similar approach, Zhu, Lu and coworkers reported the elaboration of polyzwitterionic nanoparticles containing urate oxidase or L-asparaginase in order to improve the "in vivo" circulation time and to prevent their uptake by macrophages [66]. After the step of vinylation of the amino groups of the two enzymes, the in-situ polymerization of two vinyl derivatives, a zwitterionic 2methacryloyloxyethyl phosphorylcholine and a bifunctional N, N'-methylenebisacrylamide leads to a polyzwitterionic polymer cage containing the enzyme. It appears that the urate oxidase, which is encapsulated in a crosslinked polymer shell $5 \mathrm{~nm}$ thick, retains its activity, the permeation of the urate not being hampered by steric or electrostatic constraints.

Thanks to the polyzwitterionic polymer shell, the stability of the urate oxidase nanoparticle was highly improved compared to the native enzyme. In addition, these enzyme-based nanoparticles resist the process of cell uptake in aqueous solution containing $10 \%$ serum unlike native enzymes, which are rapidly internalized by macrophages. In view of these properties, the urate oxidase-based nanoparticles injected into the blood vessels of mice exhibit markedly improved stability in vivo. It appears that a partial activity is still present after 127 hours of circulation time whereas the native enzymes completely lose their activity after 5 hours. These results highlight the key role of the nature of the polymer matrix that envelops the unique enzyme and the promising potential of these nanoparticles for therapeutic applications.

A second generation of nanocapsules has been made by introducing a specific polymerizable group into the backbone of the polyacrylamide polymer surrounding a protein leading to redox and fluorescent nanoparticles [67]. Following previous work, the enzyme glucose oxidase was first chemically modified by acryloyl groups and then an in situ polymerization was carried out with a derivative of riboflavin (2'-diallyamino-ethyl flavin) and $\mathrm{N}, \mathrm{N}^{\prime}$-methylene bis-acrylamide. The formation of the polymeric redox shell around the glucose oxidase induces an increase in diameter size from $8 \mathrm{~nm}$ for the native enzyme to $30 \mathrm{~nm}$ for the resulting nanoparticle. As previously reported, the presence of the polymer via its anchoring on the protein structure reinforces the enzyme conformation and hence its activity at higher temperatures.

More importantly, the polymerized flavin groups allow efficient electron transfer between the prosthetic glucose oxidase FAD and the electrode. In addition, the flavin groups confer fluorescence on the nanocapsules. The fluorescence intensity depending on the oxidized or reduced state of the flavin groups, the latter has been elegantly used to monitor the activity of the enzyme located in the core of the nanoparticle.

Recently, a redox nanoparticle based on glucose oxidase was designed by using a more hydrophilic riboflavin derivative functionalized with a vinyl group. Its polymerization around the enzyme carrying on average 12 acryloyl groups in presence of acrylamide derivatives provides a redox polymer shell 
around a single enzyme [68]. The resulting redox nanocapsule provides a catalytic oxidation peak at $0.4 \mathrm{~V} \mathrm{vs} \mathrm{Ag} / \mathrm{AgCl}$ in presence of glucose indicating an excellent wiring of the enzyme by the flavinbased polymer. The adsorption of these redox nanoparticles on a glassy carbon electrode modified by a deposit of multi-walled carbon nanotubes leads to an amperometric glucose biosensor without interference but with a low detection limit of $0.13 \mathrm{mmolL}^{-1}$. Its application in the design of biofuel cells in combination with a biocathode based on bilirubin oxidase and ABTS as redox mediator leads to interesting performances in terms of stability and power, a maximum power density of $1 \mathrm{~mW} \mathrm{~cm}$ being recorded at $0.378 \mathrm{~V}$. However, it should be noted that the operation of this biofuel cell requires an anode compartment saturated with $\mathrm{N}_{2}$ while the cathodic compartment contains an oxygen saturated solution. Table 1 shows the performance of some amperometric biosensors and biofuel cells prepared by immobilization of redox nanoparticles based on glucose oxidase or regular glucose oxidase in different electrode materials. The comparison of the performances obtained in term of glucose sensitivity, power density and open circuit voltage for identical quantities of glucose oxidase clearly shows the advantage of using redox nanoparticles.

Table 2: Comparison of immobilized redox enzymatic nanocapsules and regular glucose oxidase (GOX) immobilized in different materials in terms of biosensor sensitivity to glucose and power density and open circuit voltage (OCV) of biofuel cell (BFC).

\begin{tabular}{|c|c|c|c|c|c|}
\hline Material/system & $\begin{array}{c}\text { Immobilized } \\
\text { GOX amount }\end{array}$ & $\begin{array}{c}\text { Biosensor sensitivity } \\
\left(\mathrm{mA}^{-1} \mathbf{m o l L}^{-1} \mathrm{~cm}^{-2}\right)\end{array}$ & $\begin{array}{c}\text { BFC power } \\
\text { density } \\
\left(\mathrm{mWcm}^{-2}\right)\end{array}$ & $\begin{array}{c}\text { OCV } \\
\text { (V) }\end{array}$ & ref \\
\hline Nanocapsule & $100 \mu \mathrm{g}$ & 63.69 & 1.01 & 0.9 & [68] \\
\hline $\begin{array}{c}\text { MWCNT } \\
\text { compression/cellulose }\end{array}$ & $50 \mathrm{mg}$ & - & 1.3 & 0.93 & [69] \\
\hline $\begin{array}{l}\text { MWCNT-naphthoquinone } \\
\text { compression/polymers }\end{array}$ & $15 \mathrm{mg}$ & - & 1.54 & 0.76 & [70] \\
\hline $\begin{array}{l}\text { MWCNT-naphthoquinone } \\
\text { compression (flow-through) }\end{array}$ & $15 \mathrm{mg}$ & - & 1.03 & 0.66 & [71] \\
\hline $\begin{array}{l}\text { Spray-freezing reduced } \\
\text { graphene oxide }\end{array}$ & $200 \mu \mathrm{g}$ & - & 0.38 & 0.85 & [72] \\
\hline SWCNT-pyrene-biotin/avidin & $20 \mu \mathrm{g}$ & 1.6 & - & - & [73] \\
\hline $\begin{array}{c}\text { Polypyrrole- } \\
\text { adamantane//Bcyclodextrin }\end{array}$ & $0.5 \mu \mathrm{g}$ & 0.175 & - & - & [61] \\
\hline $\begin{array}{c}\mathrm{Au} / \mathrm{MXene} \text { (metal carbide) } \\
\text { nanocomposite }\end{array}$ & $320 \mu \mathrm{g}$ & 4.2 & - & - & [74] \\
\hline
\end{tabular}

The immobilization of nanoparticles on a surface with preservation of their spherical form could be exploited to develop multifunctional systems associating redox particles with particle-enzymes. These systems could lead to more efficient bioelectrochemical interfaces due to the porosity of the structure compared to layer-by-layer systems. The development of redox and enzymatic nanoparticles should make it possible to create a new generation of biofuel cells based on enzymatic cascade reactions. In addition, replacing the hydrophobic polystyrene part with a polycaprolactone in the structure of the copolymer would lead to totally bio-based and therefore biodegradable nanoparticles, thus opening the way to single-use biosensors and biofuel cells.

\section{Polymer-nanocarbon materials}

Since the discovery of $C_{60}$ in 1985 [75], carbon nanomaterials are intensively studied for any type of applications and therein biosensors since their particular optical and electronic properties provide more than just an enhanced specific surface $[76,77]$. The most investigated structures in this research topic are fullerenes [78] and carbon quantum dots [79], carbon nanotubes [80], and graphene [81] . 
Despite the electrochemical properties, $\mathrm{C}_{60}$ fullerenes did not become a privileged material in biosensing applications related to the inappropriate redox potential, little solubility and the need of sophisticated processing [82]. Relatively new and promising members of the OD nanocarbon family are carbon quantum dots. As their inorganic counterpart, these carbon dots have specific optical properties and are therefore used for optical transductions. As already discussed in the corresponding section, imprinted polymers are more and more used in combination with carbon quantum dots for biosensing applications [83, 84]. Demir et al. synthesized N-doped carbon quantum dots and used the light emission to photopolymerize the functional monomer 4acrylamidophenyl(amino)methan-iminium acetate in presence of glucuronic acid, an epitope of hyaluronan which is a biomarker for certain cancers [84]. This setup allowed quantitative imaging of cancer cells and could distinguish between healthy and tumor cells. The authors could further demonstrate no cytotoxicity of the N-doped quantum dots when applied to HaCaT cells.

Carbon nanotubes (CNTs) were considered as the carbon nanomaterial of choice for biosensors until graphene became more fashioned [85]. Related to the excellent electronic properties, CNTs are mostly used for electrochemical biosensing but examples of CNT based optical and gravimetric biosensors rely on other properties than conductivity or specific surface [80]. In combination with polymers, very efficient is the electrogeneration of thin polymer films out of functional monomers on CNTs for efficient immobilization of the receptor unit [86]. Such functions can be activated esters for covalent immobilization or host-guest units for non-covalent grafting [87]. For enzymatic biosensors, the analyte is in most cases a small organic molecule like glucose or a pesticide. An efficient method to immobilize enzymes can be the entrapment in an electrogenerated polymer matrix which remains permeable for the analyte [88]. Beside the conductivity enhancement of carbon nanotube doped polymers [89], electrochemical biosensors using the molecular imprint technique are more and more developed [90]. As an example, Kewarin et al. developed an electrochemical biosensor toward cardiac troponin $\mathrm{T}$ by imprinting this protein biomarker during electropolymerization of aniline [91]. Methylene blue was electrodeposited as redox probe on multiwalled carbon nanotubes before the formation of imprinted polyaniline (Figure 4). The differential pulse voltammetric signal of polymethylene blue decreased linearly with increasing cardiac troponin $T$ concentrations. This phenomenon is explained by the passivation of the electrode attenuating the polymethylene blue signal during rebinding of cardiac troponin $\mathrm{T}$. The reliability of this approach was confirmed with measurement in human blood plasma where the detection limit and linear range are in a realistic range. Parallel experiments with non-imprinted polyaniline further demonstrated the efficiency of this setup.

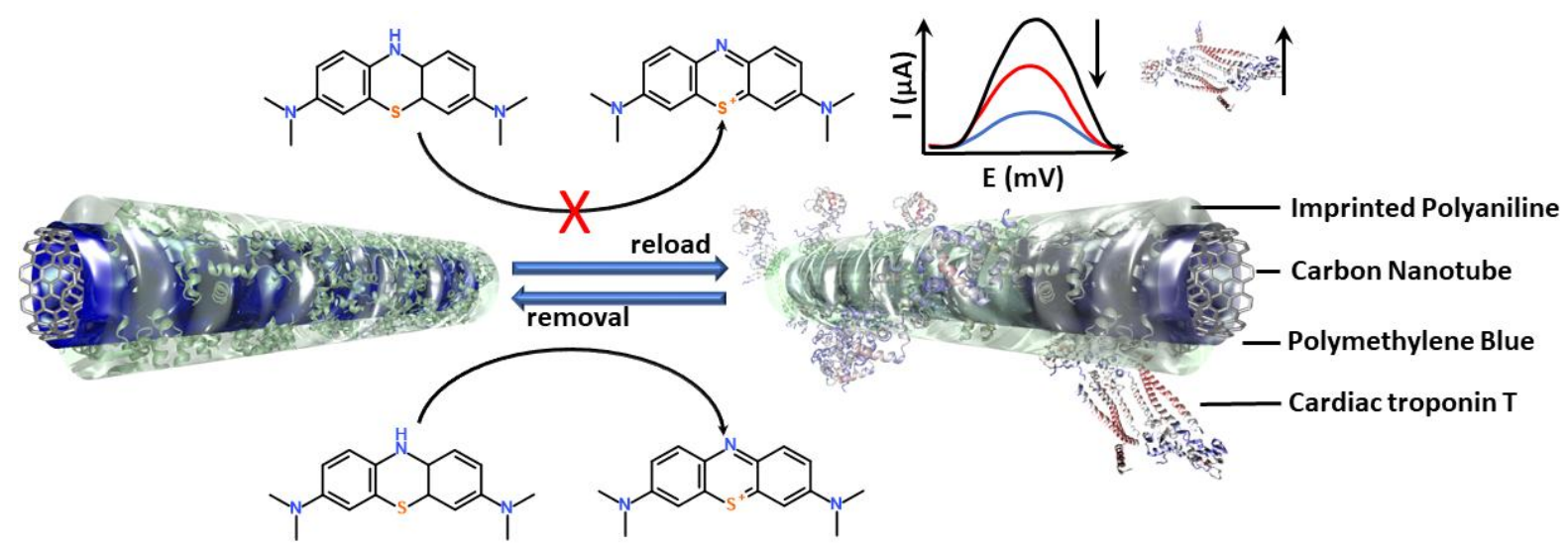

Figure 4: Sketch of polymethylene blue modified carbon nanotube electrodes with a cardiac troponin $\mathrm{T}$ imprinted polyaniline coating. The recognition of the analyte provokes a passivation of the electrode surface and lowers linearly with the cardiac troponin T concentration the polymethylene blue signal. 
The scientific interest in graphene, related 2D carbon and composites widely overpassed all other carbon nanomaterials for biosensing applications [92-94] due to their electronic and optical properties, and inexpensive availability [95]. Graphene and related materials are commonly used with other nanomaterials to form biosensors [93]. Within the vast number of examples about graphene composites [96] An original approach was applied to construct an impedimetric biosensors for the detection, and classification of the dengue virus [97]. A copolymer was formed using acrylamide, methacrylic acid, methylmethacrylate, and $\mathrm{N}$-vinylpyrrolidone with $\mathrm{N}, \mathrm{N}-(1,2-$ dihydroxyethylene) bisacrylamide as cross linker in presence of graphene oxide and a specific dengue virus serotype. The conductive imprinted polymer allowed to discriminate the subtypes of the dengue virus.

\section{Conclusion}

Polymer nanocomposites became essential components in biosensor research. The advantage is that relatively simple but reliable processes can be applied to form highly performing tools for health monitoring. The nanosized components with their specific properties mostly confer to the device higher performances but can also lead to new and efficient transduction, which cannot be obtained with macroscopic compounds. The high scientific activities around this material can be expressed with around 2000 research articles in the last twenty years (source: web of science, keywords: nanocomposites and biosensor). This technology even became of interest for commercialised devices where a couple of nanobiosensors are already in clinical use [98] Here, some recent original examples are summarized since there are already excellent exhaustive review articles about the different nanocomposites for health care biosensing [99-101].

\section{Acknowledgments}

The authors thank the French National Research Agency in the framework of the ANR-18-CE09-002201 and CBH-EUR-GS (ANR-17-EURE-0003).

\section{References}

[1] E. Gonzalez, R. Peña, A. Avila, D. Munoz-Rodriguez, Chapter 10 - Applications to Improve the Assistance of First Aiders in Outdoor Scenarios, in: M. Wister, P. Pancardo, F. Acosta, J.A. Hernández (Eds.), Intelligent Data Sensing and Processing for Health and Well-Being Applications, Academic Press2018, pp. 175-96.

[2] S.K. Vashist, J.H.T. Luong, An Overview of Point-of-Care Technologies Enabling Next-Generation Healthcare Monitoring and Management, Point-of-Care Technologies Enabling Next-Generation Healthcare Monitoring and Management, Springer International Publishing, Cham, 2019, pp. 1-25. [3] P. Bhattarai, S. Hameed, Basics of Biosensors and Nanobiosensors, in: A. Wu, W.S. Khan (Eds.), Nanobiosensors: From Design to Applications, Wiley-VCH2020, pp. 1-22.

[4] P. Mohankumar, J. Ajayan, T. Mohanraj, R. Yasodharan, Recent developments in biosensors for healthcare and biomedical applications: A review, Measurement, 167(2021) 108293.

[5] P. Bollella, L. Gorton, Enzyme based amperometric biosensors, Current Opinion in Electrochemistry, 10(2018) 157-73.

[6] J. Zhang, J. Zhao, Chapter 5 - Immuno-Biosensor, in: G. Li (Ed.) Nano-Inspired Biosensors for Protein Assay with Clinical Applications, Elsevier2019, pp. 115-37. 
[7] A. Kowalczyk, Trends and perspectives in DNA biosensors as diagnostic devices, Current Opinion in Electrochemistry, 23(2020) 36-41.

[8] L.A. Stanciu, Q. Wei, A.K. Barui, N. Mohammad, Recent Advances in Aptamer-Based Biosensors for Global Health Applications, Annual Review of Biomedical Engineering, 23(2021) null.

[9] M.T. Stumpp, P. Amstutz, DARPins: a true alternative to antibodies, Curr Opin Drug Discov Devel, 10(2007) 153-9.

[10] S.J. Updike, G.P. Hicks, The Enzyme Electrode, Nature, 214(1967) 986-8.

[11] J.J. Long, A.M. Benoudjit, F.A. Arris, F. Ali, W.W.A. Wan Salim, Polymers in Biosensors, in: A. Amid, S. Sulaiman, D.N. Jimat, N.F.M. Azmin (Eds.), Multifaceted Protocol in Biotechnology, Springer Singapore, Singapore, 2018, pp. 151-65.

[12] S. Ramanavicius, A. Ramanavicius, Conducting Polymers in the Design of Biosensors and Biofuel Cells, Polymers (Basel), 13(2020).

[13] S. Cosnier, M. Holzinger, Electrosynthesized polymers for biosensing, Chem Soc Rev, 40(2011) 2146-56.

[14] M. Pirzada, Z. Altintas, Nanomaterials for Healthcare Biosensing Applications, Sensors (Basel), 19(2019).

[15] M. Holzinger, A. Le Goff, S. Cosnier, Nanomaterials for biosensing applications: A Review, Front Chem, 2(2014) 63.

[16] A.B. Asha, R. Narain, Chapter 15 - Nanomaterials properties, in: R. Narain (Ed.) Polymer Science and Nanotechnology, Elsevier2020, pp. 343-59.

[17] E. Boisselier, D. Astruc, Gold nanoparticles in nanomedicine: preparations, imaging, diagnostics, therapies and toxicity, Chemical Society Reviews, 38(2009) 1759.

[18] L.L. Tayo, Stimuli-responsive nanocarriers for intracellular delivery, Biophysical Reviews, 9(2017) 931-40.

[19] A.A. Yaqoob, H. Ahmad, T. Parveen, A. Ahmad, M. Oves, I.M.I. Ismail, et al., Recent Advances in Metal Decorated Nanomaterials and Their Various Biological Applications: A Review, Front Chem, $8(2020) 341$.

[20] S.A. Akintelu, S.C. Olugbeko, A.S. Folorunso, A review on synthesis, optimization, characterization and antibacterial application of gold nanoparticles synthesized from plants, International Nano Letters, 10(2020) 237-48.

[21] T. Hassan, A. Salam, A. Khan, S.U. Khan, H. Khanzada, M. Wasim, et al., Functional nanocomposites and their potential applications: A review, Journal of Polymer Research, 28(2021). [22] S. Fu, Z. Sun, P. Huang, Y. Li, N. Hu, Some basic aspects of polymer nanocomposites: A critical review, Nano Materials Science, 1(2019) 2-30. 
[23] S. Komarneni, Feature article. Nanocomposites, Journal of Materials Chemistry, 2(1992) 121930.

[24] D.W. Hoffman, R. Roy, S. Komarneni, Diphasic Xerogels, A New Class of Materials: Phases in the System Al2o3-Sio2, Journal of the American Ceramic Society, 67(1984) 468-71.

[25] S. Kumar, Sarita, M. Nehra, N. Dilbaghi, K. Tankeshwar, K.-H. Kim, Recent advances and remaining challenges for polymeric nanocomposites in healthcare applications, Progress in Polymer Science, 80(2018) 1-38.

[26] S. Shrivastava, N. Jadon, R. Jain, Next-generation polymer nanocomposite-based electrochemical sensors and biosensors: A review, TrAC Trends in Analytical Chemistry, 82(2016) 55-67.

[27] G. Wang, A. Morrin, M. Li, N. Liu, X. Luo, Nanomaterial-doped conducting polymers for electrochemical sensors and biosensors, J Mater Chem B, 6(2018) 4173-90.

[28] H. Weller, Colloidal Semiconductor Q-Particles: Chemistry in the Transition Region Between Solid State and Molecules, Angewandte Chemie International Edition in English, 32(1993) 41-53.

[29] A.P. Litvin, I.V. Martynenko, F. Purcell-Milton, A.V. Baranov, A.V. Fedorov, Y.K. Gun'ko, Colloidal quantum dots for optoelectronics, Journal of Materials Chemistry A, 5(2017) 13252-75.

[30] A.M. Wagner, J.M. Knipe, G. Orive, N.A. Peppas, Quantum dots in biomedical applications, Acta Biomaterialia, 94(2019) 44-63.

[31] V.G. Reshma, P.V. Mohanan, Quantum dots: Applications and safety consequences, Journal of Luminescence, 205(2019) 287-98.

[32] H. Labiadh, S. Hidouri, ZnS quantum dots and their derivatives: Overview on identity, synthesis and challenge into surface modifications for restricted applications, Journal of King Saud University Science, 29(2017) 444-50.

[33] X. Wang, Y. Feng, P. Dong, J. Huang, A Mini Review on Carbon Quantum Dots: Preparation, Properties, and Electrocatalytic Application, Front Chem, 7(2019) 671.

[34] X.T. Zheng, A. Ananthanarayanan, K.Q. Luo, P. Chen, Glowing Graphene Quantum Dots and Carbon Dots: Properties, Syntheses, and Biological Applications, Small, 11(2015) 1620-36.

[35] L. Dyadyusha, H. Yin, S. Jaiswal, T. Brown, J.J. Baumberg, F.P. Booy, et al., Quenching of CdSe quantum dot emission, a new approach for biosensing, Chemical Communications, (2005) 3201-3.

[36] H. Dong, W. Gao, F. Yan, H. Ji, H. Ju, Fluorescence Resonance Energy Transfer between Quantum Dots and Graphene Oxide for Sensing Biomolecules, Analytical Chemistry, 82(2010) 5511-7.

[37] M. Diaz-Alvarez, A. Martin-Esteban, Molecularly Imprinted Polymer-Quantum Dot Materials in Optical Sensors: An Overview of Their Synthesis and Applications, Biosensors (Basel), 11(2021). [38] R. Li, Y. Feng, G. Pan, L. Liu, Advances in Molecularly Imprinting Technology for Bioanalytical Applications, Sensors, 19(2019) 177. 
[39] Q. Yang, J. Li, X. Wang, H. Peng, H. Xiong, L. Chen, Strategies of molecular imprinting-based fluorescence sensors for chemical and biological analysis, Biosensors and Bioelectronics, 112(2018) 54-71.

[40] X. Wang, J. Yu, J. Li, Q. Kang, D. Shen, L. Chen, Quantum dots based imprinting fluorescent nanosensor for the selective and sensitive detection of phycocyanin: A general imprinting strategy toward proteins, Sensors and Actuators B: Chemical, 255(2018) 268-74.

[41] G. Mo, D. Qin, X. Jiang, X. Zheng, W. Mo, B. Deng, A sensitive electrochemiluminescence biosensor based on metal-organic framework and imprinted polymer for squamous cell carcinoma antigen detection, Sensors and Actuators B: Chemical, 310(2020) 127852.

[42] D.Y. Li, Y.P. Qin, H.Y. Li, X.W. He, W.Y. Li, Y.K. Zhang, A "turn-on" fluorescent receptor for detecting tyrosine phosphopeptide using the surface imprinting procedure and the epitope approach, Biosens Bioelectron, 66(2015) 224-30.

[43] N. Saeedzadeh Amiri, M.-R. Milani Hosseini, Application of ratiometric fluorescence sensorbased microwave-assisted synthesized CdTe quantum dots and mesoporous structured epitopeimprinted polymers for highly efficient determination of tyrosine phosphopeptide, Analytical Methods, 12(2020) 63-72.

[44] Y. Li, H. Schluesener, S. Xu, Gold nanoparticle-based biosensors, Gold Bull, 43(2010) 29-41.

[45] N. Elahi, M. Kamali, M.H. Baghersad, Recent biomedical applications of gold nanoparticles: A review, Talanta, 184(2018) 537-56.

[46] L. Qin, G. Zeng, C. Lai, D. Huang, P. Xu, C. Zhang, et al., "Gold rush" in modern science: Fabrication strategies and typical advanced applications of gold nanoparticles in sensing, Coordination Chemistry Reviews, 359(2018) 1-31.

[47] V. Amendola, R. Pilot, M. Frasconi, O.M. Maragò, M.A. latì, Surface plasmon resonance in gold nanoparticles: a review, Journal of Physics: Condensed Matter, 29(2017) 203002.

[48] S. Dutta, K. Saikia, P. Nath, Smartphone based LSPR sensing platform for bio-conjugation detection and quantification, RSC Advances, 6(2016) 21871-80.

[49] P.K. Jain, W. Huang, M.A. El-Sayed, On the Universal Scaling Behavior of the Distance Decay of Plasmon Coupling in Metal Nanoparticle Pairs: A Plasmon Ruler Equation, Nano Letters, 7(2007) 2080-8.

[50] M. Lu, H. Zhu, C.G. Bazuin, W. Peng, J.F. Masson, Polymer-Templated Gold Nanoparticles on Optical Fibers for Enhanced-Sensitivity Localized Surface Plasmon Resonance Biosensors, ACS Sens, 4(2019) 613-22.

[51] K. Avelino, L.S. Oliveira, N. Lucena-Silva, C.A.S. Andrade, M.D.L. Oliveira, Flexible sensor based on conducting polymer and gold nanoparticles for electrochemical screening of HPV families in cervical specimens, Talanta, 226(2021) 122118. 
[52] T. Vural, Y.T. Yaman, S. Ozturk, S. Abaci, E.B. Denkbas, Electrochemical immunoassay for detection of prostate specific antigen based on peptide nanotube-gold nanoparticle-polyaniline immobilized pencil graphite electrode, J Colloid Interface Sci, 510(2018) 318-26.

[53] F. Fang, M. Li, J. Zhang, C.-S. Lee, Different Strategies for Organic Nanoparticle Preparation in Biomedicine, ACS Materials Letters, 2(2020) 531-49.

[54] Y. Zhang, F. Fang, L. Li, J. Zhang, Self-Assembled Organic Nanomaterials for Drug Delivery, Bioimaging, and Cancer Therapy, ACS Biomaterials Science \& Engineering, 6(2020) 4816-33.

[55] Y. Miura, Y. Hoshino, H. Seto, Glycopolymer Nanobiotechnology, Chem Rev, 116(2016) 1673-92.

[56] I. Pramudya, H. Chung, Recent progress of glycopolymer synthesis for biomedical applications, Biomater Sci, 7(2019) 4848-72.

[57] D.D. Gadade, S.S. Pekamwar, Cyclodextrin Based Nanoparticles for Drug Delivery and Theranostics, Adv Pharm Bull, 10(2020) 166-83.

[58] A.J. Gross, X. Chen, F. Giroud, C. Travelet, R. Borsali, S. Cosnier, Redox-Active Glyconanoparticles as Electron Shuttles for Mediated Electron Transfer with Bilirubin Oxidase in Solution, J Am Chem Soc, 139(2017) 16076-9.

[59] A.J. Gross, R. Haddad, C. Travelet, E. Reynaud, P. Audebert, R. Borsali, et al., Redox-Active Carbohydrate-Coated Nanoparticles: Self-Assembly of a Cyclodextrin-Polystyrene Glycopolymer with Tetrazine-Naphthalimide, Langmuir, 32(2016) 11939-45.

[60] J.L. Hammond, A.J. Gross, F. Giroud, C. Travelet, R. Borsali, S. Cosnier, Solubilized Enzymatic Fuel Cell (SEFC) for Quasi-Continuous Operation Exploiting Carbohydrate Block Copolymer Glyconanoparticle Mediators, ACS Energy Lett, 4(2019) 142-8.

[61] M. Holzinger, L. Bouffier, R. Villalonga, S. Cosnier, Adamantane/ $\beta$-cyclodextrin affinity biosensors based on single-walled carbon nanotubes, Biosensors and Bioelectronics, 24(2009) 1128-34.

[62] M. Holzinger, M. Singh, S. Cosnier, Biotin - $\beta$-cyclodextrin: a new host-guest system for the immobilization of biomolecules, Langmuir, 28(2012) 12569-74.

[63] I. Otsuka, M. Osaka, Y. Sakai, C. Travelet, J.-L. Putaux, R. Borsali, Self-Assembly of Maltoheptaose-block-Polystyrene into Micellar Nanoparticles and Encapsulation of Gold Nanoparticles, Langmuir, 29(2013) 15224-30.

[64] M. Carriere, P.H.M. Buzzetti, K. Gorgy, M. Mumtaz, C. Travelet, R. Borsali, et al., Functionalizable Glyconanoparticles for a Versatile Redox Platform, Nanomaterials (Basel), 11(2021).

[65] M. Yan, J. Du, Z. Gu, M. Liang, Y. Hu, W. Zhang, et al., A novel intracellular protein delivery platform based on single-protein nanocapsules, Nature Nanotechnology, 5(2010) 48-53.

[66] X. Zhang, W. Chen, X. Zhu, Y. Lu, Encapsulating Therapeutic Proteins with Polyzwitterions for Lower Macrophage Nonspecific Uptake and Longer Circulation Time, ACS Appl Mater Interfaces, 9(2017) 7972-8. 
[67] D. Chen, Y. Huang, H. Jiang, W. Yasen, D. Guo, Y. Su, et al., Fabrication of Activity-Reporting Glucose Oxidase Nanocapsules with Oxygen-Independent Fluorescence Variation, ACS Appl Mater Interfaces, 10(2018) 26005-15.

[68] J. Huang, Y. Zhang, F. Ding, D. Chen, Y. Wang, X. Jin, et al., Rational design of electroactive redox enzyme nanocapsules for high-performance biosensors and enzymatic biofuel cell, Biosens Bioelectron, 174(2021) 112805.

[69] A. Zebda, C. Gondran, A. Le Goff, M. Holzinger, P. Cinquin, S. Cosnier, Mediatorless high-power glucose biofuel cells based on compressed carbon nanotube-enzyme electrodes, Nature Communications, 2(2011) 370.

[70] B. Reuillard, A. Le Goff, C. Agnes, M. Holzinger, A. Zebda, C. Gondran, et al., High power enzymatic biofuel cell based on naphthoquinone-mediated oxidation of glucose by glucose oxidase in a carbon nanotube 3D matrix, Physical Chemistry Chemical Physics, 15(2013) 4892-6.

[71] C. Abreu, Y. Nedellec, A.J. Gross, O. Ondel, F. Buret, A.L. Goff, et al., Assembly and Stacking of Flow-through Enzymatic Bioelectrodes for High Power Glucose Fuel Cells, ACS Appl Mater Interfaces, 9(2017) 23836-42.

[72] J. Lee, K. Hyun, J.M. Park, H.S. Park, Y. Kwon, Maximizing the enzyme immobilization of enzymatic glucose biofuel cells through hierarchically structured reduced graphene oxide, International Journal of Energy Research, (2021) in press (doi: 10.1002/er.7155).

[73] M. Holzinger, R. Haddad, A. Maaref, S. Cosnier, Amperometric biosensors based on biotinlylated single-walled carbon nanotubes, J Nanosci Nanotechnol, 9(2009) 6042-6.

[74] R.B. Rakhi, P. Nayak, C. Xia, H.N. Alshareef, Novel amperometric glucose biosensor based on MXene nanocomposite, Sci Rep, 6(2016) 36422.

[75] H.W. Kroto, J.R. Heath, S.C. O'Brien, R.F. Curl, R.E. Smalley, C60: Buckminsterfullerene, Nature, 318(1985) 162-3.

[76] T. Pasinszki, M. Krebsz, T.T. Tung, D. Losic, Carbon Nanomaterial Based Biosensors for NonInvasive Detection of Cancer and Disease Biomarkers for Clinical Diagnosis, Sensors (Basel), 17(2017) E1919.

[77] Z. Wang, Z. Dai, Carbon nanomaterial-based electrochemical biosensors: an overview, Nanoscale, 7(2015) 6420-31.

[78] S. Pilehvar, K. De Wael, Recent Advances in Electrochemical Biosensors Based on Fullerene- $\mathrm{C}_{60}$ Nano-Structured Platforms, Biosensors, 5(2015) 712-35.

[79] S. Sahin, C. Unlu, L. Trabzon, Affinity biosensors developed with quantum dots in microfluidic systems, Emergent Mater, (2021) 1-23.

[80] M. Sireesha, V. Jagadeesh Babu, A.S. Kranthi Kiran, S. Ramakrishna, A review on carbon nanotubes in biosensor devices and their applications in medicine, Nanocomposites, 4(2018) 36-57. 
[81] J. Peña-Bahamonde, H.N. Nguyen, S.K. Fanourakis, D.F. Rodrigues, Recent advances in graphenebased biosensor technology with applications in life sciences, Journal of Nanobiotechnology, 16(2018) 75.

[82] A. Bianco, Y. Chen, E. Frackowiak, M. Holzinger, N. Koratkar, V. Meunier, et al., Carbon science perspective in 2020: Current research and future challenges, Carbon, 161(2020) 373-91.

[83] K. Haupt, K. Mosbach, Molecularly Imprinted Polymers and Their Use in Biomimetic Sensors, Chemical Reviews, 100(2000) 2495-504.

[84] B. Demir, M.M. Lemberger, M. Panagiotopoulou, P.X. Medina Rangel, S. Timur, T. Hirsch, et al., Tracking Hyaluronan: Molecularly Imprinted Polymer Coated Carbon Dots for Cancer Cell Targeting and Imaging, ACS Appl Mater Interfaces, 10(2018) 3305-13.

[85] N. Yang, X. Chen, T. Ren, P. Zhang, D. Yang, Carbon nanotube based biosensors, Sensors and Actuators B: Chemical, 207, Part A(2015) 690-715.

[86] S. Cosnier, M. Holzinger, A. Le Goff, Conductive Polymers, Immobilization of Macromolecular Bio-Entities, in: G. Kreysa, K.-i. Ota, R. Savinell (Eds.), Encyclopedia of Applied Electrochemistry, Springer New York2014, pp. 253-60.

[87] M. Holzinger, A. Le Goff, S. Cosnier, Supramolecular immobilization of bio-entities for bioelectrochemical applications, New J Chem, 38(2014) 5173-80.

[88] W.H. Scouten, J.H.T. Luong, R. Stephen Brown, Enzyme or protein immobilization techniques for applications in biosensor design, Trends in Biotechnology, 13(1995) 178-85.

[89] S. Cosnier, R.E. Ionescu, M. Holzinger, Aqueous dispersions of SWCNTs using pyrrolic surfactants for the electro-generation of homogeneous nanotube-composites. Application to the design of amperometric biosensor, J Mater Chem, 18(2008) 5129-33.

[90] H. Pandey, P. Khare, S. Singh, S.P. Singh, Carbon nanomaterials integrated molecularly imprinted polymers for biological sample analysis: A critical review, Materials Chemistry and Physics, 239(2020) 121966.

[91] K. Phonklam, R. Wannapob, W. Sriwimol, P. Thavarungkul, T. Phairatana, A novel molecularly imprinted polymer PMB/MWCNTs sensor for highly-sensitive cardiac troponin T detection, Sensors and Actuators B: Chemical, 308(2020) 127630.

[92] N. Celik, W. Balachandran, N. Manivannan, Graphene-based biosensors: methods, analysis and future perspectives, IET Circuits, Devices \&amp; Systems, Institution of Engineering and Technology, Stevenage, UK, 2015, pp. 434-45.

[93] S.K. Krishnan, E. Singh, P. Singh, M. Meyyappan, H.S. Nalwa, A review on graphene-based nanocomposites for electrochemical and fluorescent biosensors, RSC Advances, 9(2019) 8778-881. 
[94] J. Muñoz, L.J. Brennan, F. Céspedes, Y.K. Gun'ko, M. Baeza, Characterization protocol to improve the electroanalytical response of graphene-polymer nanocomposite sensors, Composites Science and Technology, 125(2016) 71-9.

[95] P. Suvarnaphaet, S. Pechprasarn, Graphene-Based Materials for Biosensors: A Review, Sensors (Basel), 17(2017) 2161.

[96] W.K. Chee, H.N. Lim, N.M. Huang, I. Harrison, Nanocomposites of graphene/polymers: a review, RSC Advances, 5(2015) 68014-51.

[97] K. Navakul, C. Warakulwit, P.T. Yenchitsomanus, A. Panya, P.A. Lieberzeit, C. Sangma, A novel method for dengue virus detection and antibody screening using a graphene-polymer based electrochemical biosensor, Nanomedicine, 13(2017) 549-57.

[98] A. Dedeoglu, S.I. Kaya, N.K. Bakirhan, S.A. Ozkan, Chapter Twelve - Nanotechnological approaches and materials in commercial biosensors, in: M.K. Sezgintürk (Ed.) Commercial Biosensors and Their Applications, Elsevier2020, pp. 301-53.

[99] K. Omidfar, A. Ahmadi, L. Syedmoradi, S.M. Khoshfetrat, B. Larijani, Point-of-care biosensors in medicine: a brief overview of our achievements in this field based on the conducted research in EMRI (endocrinology and metabolism research Institute of Tehran University of medical sciences) over the past fourteen years, J Diabetes Metab Disord, (2020) 1-5.

[100] Y. Lu, M.C. Biswas, Z. Guo, J.W. Jeon, E.K. Wujcik, Recent developments in bio-monitoring via advanced polymer nanocomposite-based wearable strain sensors, Biosens Bioelectron, 123(2019) 167-77.

[101] A.A. D'Souza, D. Kumari, R. Banerjee, Nanocomposite biosensors for point-of-care-evaluation of food quality and safety, in: A.M. Grumezescu (Ed.) Nanobiosensors, Academic Press2017, pp. 62976. 